\title{
A COUPLED SYSTEM OF FUNCTIONAL DIFFERENTIAL EQUATIONS IN REFLEXIVE BANACH SPACES
}

\author{
A.M.A. El-Sayed ${ }^{1}$, W.G. El-Sayed ${ }^{2}$, A.A.H. Abd El-Mowla ${ }^{3} \S$ \\ ${ }^{1,2}$ Faculty of Science \\ Alexandria University \\ Alexandria, EGYPT \\ ${ }^{3}$ Faculty of Science \\ Omar Al-Mukhtar University \\ Derna, LIBYA
}

\begin{abstract}
We present an existence theorem for at least one weak solution for the coupled system of functional differential equations

$$
\begin{aligned}
& x^{\prime}(t)=f_{1}\left(t, y^{\prime}(t)\right), t \in(0, T], \\
& y^{\prime}(t)=f_{2}\left(t, x^{\prime}(t)\right), t \in(0, T]
\end{aligned}
$$

in reflexive Banach spaces.

AMS Subject Classification: 35D30, 34Gxx

Key Words: weak solution, functional differential equations, O'Regan fixed point theorem, coupled system
\end{abstract}

\section{Introduction and Preliminaries}

Let $E$ be a reflexive Banach space with norm $\|\cdot\|$ and dual $E^{*}$, and $L^{1}(I)$ be the space of Lebesgue integrable functions on the interval $I=[0, T]$. Denote by $C[I, E]$ the Banach space of strongly continuous functions $x: I \rightarrow E$ with sup-norm $\|\cdot\|_{0}$.

Received: $\quad$ October 8, 2016

Revised: December 7, 2016

Published: February 28, 2017

${ }^{\S}$ Correspondence author (c) 2017 Academic Publications, Ltd. url: www.acadpubl.eu 
Such systems appear in many problems of applied nature (see [1],[3], [4], [9][11] and [13]). Su [18] discussed a two-point boundary value problem for a coupled system of fractional differential equations. Gafiychuk et al. [11] analyzed the solutions of coupled nonlinear fractional reaction-diffusion equations. The solvability of the coupled systems of integral equations in reflexive Banach space was proved in (see [5]-[7]).

In [15], O'Regan studied, in the reflexive Banach space, the existence of weak solutions of the initial value problem

$$
\frac{d}{d t} x(t)=f(t, x(t)), \quad t \in(0, T] .
$$

Recently, the authors studied the existence of weak solutions of the initial value problem

$$
\frac{d}{d t} x(t)=f\left(t, \frac{d}{d t} x(t)\right), \quad t \in(0, T] .
$$

in reflexive and nonreflexive Banach spaces (see [8]).

In this paper we study the existence of weak solutions for the coupled system of the functional differential equations

$$
\begin{aligned}
& \frac{d x}{d t}=f_{1}\left(t, \frac{d y}{d t}\right), x(0)=x_{0}, t \in(0, T] \\
& \frac{d y}{d t}=f_{2}\left(t, \frac{d x}{d t}\right), y(0)=y_{0}, t \in(0, T]
\end{aligned}
$$

in the reflexive Banach space $E$. For this aim we study, firstly, the existence of weak solutions for the coupled system of the functional equations

$$
\begin{aligned}
& u(t)=f_{1}(t, v(t)), t \in I \\
& v(t)=f_{2}(t, u(t)), t \in I
\end{aligned}
$$

in the reflexive Banach space $E$.

Now, we shall present some auxiliary results that will be needed in this work.

(1) A function $h: E \rightarrow E$ is said to be weakly Lipschitz if for every $\phi \in$ $E^{*}$ there exists a positive constant $K$ such that

$$
\phi(h(x(.))-h(y(.))) \leq K \phi(x(.)-y(.)) .
$$

(2) $h($.$) is said to be weakly continuous if for every \phi \in E^{*}, \phi(h()$. is continuous see ([12] and [14]). 
(3) A function $h: E \rightarrow E$ is said to be weakly sequentially continuous if $h$ maps weakly convergent sequences in $E$ to weakly convergent sequences in $E$.

It is clear that (1) implies (2) and (2) implies (3). If $h$ linear, then (2) and (3) are equivalent. The relation between weak and weak sequentially continuous of mapping is studied in details in [2].

Now, we have the following theorem due to Rubin (see [16]) and some propositions which will be used in the sequel (see [7] and [17]).

Theorem 1. If $E$ is metrizable (i.e., the topology is induced by a metric). Then the weakly sequentially continuous functions are weakly continuous.

Proposition 1. A subset of a reflexive Banach space is weakly compact if and only if it is closed in the weak topology and bounded in the norm topology.

The following result follows directly from the Hahn-Banach theorem.

Proposition 2. Let $E$ be a normed space with $x_{0} \neq 0$. Then there exists a $\phi \in E^{*}$ with

$\|\phi\|=1$ and $\phi\left(x_{0}\right)=\left\|x_{0}\right\|$.

Also, we have the following fixed point theorem, due to O'Regan, in reflexive Banach space (see [14]).

Theorem 2. (O'Regan fixed point theorem) Let $E$ be a Banach space and let $Q$ be a nonempty, bounded, closed and convex subset of the space $E$ and let $F: Q \rightarrow Q$ be a weakly sequentially continuous and assume that $F Q(t)$ is relatively weakly compact in $E$ for each $t \in I$. Then, $F$ has a fixed point in the set $Q$.

\section{Coupled System of Functional Equations}

Here, we discuss the existence of weak solutions for the coupled system (3)-(4) in the reflexive Banach space $E$.

The coupled system (3)-(4) will be investigated under the following assumptions

$f_{i}: I \times E \rightarrow E, \quad i=1,2$ such that

(i) For each $u \in C[I, E], f_{i}(., u()$.$) are continuous on I$;

(ii) For each $t \in I, f_{i}(t,$.$) are weakly Lipschitz with Lipschitz constants K_{i}$, 
where $a_{i}=\sup \left\{\phi\left(f_{i}(t, 0)\right): t \in I\right\}$.

Now, let $X$ be the class of all ordered pairs $(u, v), u, v \in C[I, E]$ with the norm

$$
\|(u, v)\|=\|u\|+\|v\|
$$

Definition 1. By a solution to the coupled system (3)-(4), we mean the pair of functions $(u, v) \in X, u, v \in C[I, E]$ which satisfies (3)-(4) weakly. This is equivalent to finding $(u, v) \in X, u, v \in C[I, E]$ with

$$
\begin{aligned}
& \phi(u(t))=\phi\left(f_{1}(t, v(t))\right), t \in I \\
& \phi(v(t))=\phi\left(f_{2}(t, u(t))\right), t \in I
\end{aligned}
$$

for all $\phi \in E^{*}$.

Now, we can prove the following existence theorem.

Theorem 3. Under the assumptions (i)-(ii), the coupled system (3)-(4) has at least one weak solution $(u, v) \in X, \quad u, v \in C[I, E]$.

Proof. Define the operator $A$ by

$$
A(u(t), v(t))=\left(A_{1} v(t), A_{2} u(t)\right)
$$

where

$$
\begin{aligned}
& A_{1} v(t)=f_{1}(t, v(t)), t \in I \\
& A_{2} u(t)=f_{2}(t, u(t)), t \in I .
\end{aligned}
$$

Now, define the set $\Omega$ by

$$
\Omega=\left\{(u, v), \quad u, v \in C[I, E]:\|v\|_{0} \leq r_{1},\|u\|_{0} \leq r_{2}, r=r_{1}+r_{2}\right\} .
$$

The reminder of the proof will be given in four steps.

Step 1: The operator $A$ maps $X$ into itself. For this, let $u, v \in C[I, E]$. Let $t_{1}, t_{2} \in I, t_{2}>t_{1}$, (without loss of generality assume that $A(u, v)\left(t_{2}\right)-$ $\left.A(u, v)\left(t_{1}\right) \neq 0\right)$, then

$$
\begin{aligned}
A_{1} v\left(t_{2}\right)-A_{1} v\left(t_{1}\right) & =f_{1}\left(t_{2}, v\left(t_{2}\right)\right)-f_{1}\left(t_{1}, v\left(t_{1}\right)\right) \\
& =f_{1}\left(t_{2}, v\left(t_{2}\right)\right)-f_{1}\left(t_{1}, v\left(t_{1}\right)\right) \\
& -f_{1}\left(t_{2}, v\left(t_{1}\right)\right)+f_{1}\left(t_{2}, v\left(t_{1}\right)\right)
\end{aligned}
$$

by proposition 2 we have

$$
\left\|A_{1} v\left(t_{2}\right)-A_{1} v\left(t_{1}\right)\right\|=\phi\left(A_{1} v\left(t_{2}\right)-A_{1} v\left(t_{1}\right)\right)
$$




$$
\begin{aligned}
& \leq \phi\left(f_{1}\left(t_{2}, v\left(t_{2}\right)\right)-f_{1}\left(t_{2}, v\left(t_{1}\right)\right)\right) \\
& +\phi\left(f_{1}\left(t_{2}, v\left(t_{1}\right)\right)-f_{1}\left(t_{1}, v\left(t_{1}\right)\right)\right) \\
& \leq K_{1} \phi\left(v\left(t_{2}\right)-v\left(t_{1}\right)\right) \\
& +\phi\left(f_{1}\left(t_{2}, v\left(t_{1}\right)\right)-f_{1}\left(t_{1}, v\left(t_{1}\right)\right)\right) \\
& \leq K_{1}\left\|v\left(t_{2}\right)-v\left(t_{1}\right)\right\| \\
& +\left\|f_{1}\left(t_{2}, v\left(t_{1}\right)\right)-f_{1}\left(t_{1}, v\left(t_{1}\right)\right)\right\| .
\end{aligned}
$$

Similarity, we can show that

$$
\begin{aligned}
\left\|A_{2} u\left(t_{2}\right)-A_{2} u\left(t_{1}\right)\right\| & \leq K_{2}\left\|u\left(t_{2}\right)-u\left(t_{1}\right)\right\| \\
& +\left\|f_{2}\left(t_{2}, u\left(t_{1}\right)\right)-f_{2}\left(t_{1}, u\left(t_{1}\right)\right)\right\| .
\end{aligned}
$$

Therefore,

$$
\begin{aligned}
\left\|A(u, v)\left(t_{2}\right)-A(u, v)\left(t_{1}\right)\right\| & =\|\left(A_{1} v\left(t_{2}\right), A_{2} u\left(t_{2}\right)\right) \\
& -\left(A_{1} v\left(t_{1}\right), A_{2} u\left(t_{1}\right)\right) \| \\
& =\|\left(A_{1} v\left(t_{2}\right)-A_{1} v\left(t_{1}\right), A_{2} u\left(t_{2}\right)\right. \\
& \left.-A_{2} u\left(t_{1}\right)\right) \| \\
& =\left\|A_{1} v\left(t_{2}\right)-A_{1} v\left(t_{1}\right)\right\| \\
& \left.+\| A_{2} u\left(t_{2}\right)-A_{2} u\left(t_{1}\right)\right) \| \\
& \leq K_{1}\left\|v\left(t_{2}\right)-v\left(t_{1}\right)\right\| \\
& +\left\|f_{1}\left(t_{2}, v\left(t_{1}\right)\right)-f_{1}\left(t_{1}, v\left(t_{1}\right)\right)\right\| \\
& +K_{2}\left\|u\left(t_{2}\right)-u\left(t_{1}\right)\right\| \\
& +\left\|f_{2}\left(t_{2}, u\left(t_{1}\right)\right)-f_{2}\left(t_{1}, u\left(t_{1}\right)\right)\right\| .
\end{aligned}
$$

Which prove that $A: X \rightarrow X$.

Step 2: The operator $A$ maps $\Omega$ into itself. Let $(u, v) \in \Omega$ then by proposition 2 we have

$$
\begin{aligned}
\left\|A_{1} v(t)\right\| & =\phi\left(A_{1} v(t)\right)=\phi\left(f_{1}(t, v(t))\right) \\
& \leq \phi\left(f_{1}(t, 0)\right)+K_{1} \phi(v(t)) \\
& \leq a_{1}+K_{1}\|v\|_{0} \\
& \leq a_{1}+K_{1} r_{1} .
\end{aligned}
$$

Then

$$
\left\|A_{1} v\right\|_{0}=\sup _{t \in I}\left\|A_{1} v(t)\right\| \leq a_{1}+K_{1} r_{1}=r_{1}, r_{1}=\frac{a_{1}}{1-K_{1}}
$$


Similarity, we can show that

$$
\left\|A_{2} u\right\|_{0} \leq a_{2}+K_{2} r_{2}=r_{2}, r_{2}=\frac{a_{2}}{1-K_{2}} .
$$

Now

$$
\|A(u, v)(t)\|=\left\|\left(A_{1} v(t), A_{2} u(t)\right)\right\| \leq\left\|A_{1} v(t)\right\|+\left\|A_{2} u(t)\right\| \leq r .
$$

Then

$$
\|A(u, v)\|_{0} \leq r
$$

Hence, for any $(u, v) \in \Omega$ we have $A(u, v) \in \Omega$, consequently $A \Omega \subset \Omega \Rightarrow A$ : $\Omega \rightarrow \Omega$.

Step 3: $A \Omega(t)$ is relatively weakly compact in $E$. Note that $\Omega$ is nonempty, closed, convex and bounded subset of $X$. According to proposition 1, $A \Omega$ is bounded in $X$ and closed in weak topology, hence $A \Omega$ is relatively weakly compact in $X$ implies $A \Omega(t)$ is relatively weakly compact in $E$ for each $t \in I$.

Step 4: The operator $A$ is weakly sequentially continuous on $\Omega$. Let $\left\{V_{n}\right\}$ be sequence in $\Omega$ converges weakly to $V$, then we have the two sequences $\left\{v_{n}(t)\right\},\left\{u_{n}(t)\right\}$ converges weakly to $v(t), u(t)$, respectively for all $t \in I$. Since $f_{1}(t, v(t)), f_{2}(t, u(t))$ are weakly Lipschitz, then $f_{1}(t, v(t)), f_{2}(t, u(t))$ are weakly continuous, then we have that $f_{1}(t, v(t)), f_{2}(t, u(t))$ are weakly sequentially continuous in the second argument. Thus $f_{1}\left(t, v_{n}(t)\right), f_{2}\left(t, u_{n}(t)\right)$ converges weakly to $f_{1}(t, v(t)), f_{2}(t, u(t))$ respectively, then $\phi\left(f_{1}\left(t, v_{n}(t)\right)\right), \phi\left(f_{2}\left(t, u_{n}(t)\right)\right)$ converges strongly to $\phi\left(f_{1}(t, v(t))\right), \phi\left(f_{2}(t, u(t))\right)$ respectively. Thus

$$
\phi\left(A_{1} v_{n}(t)\right) \rightarrow \phi\left(A_{1} v(t)\right), \quad \phi\left(A_{2} u_{n}(t)\right) \rightarrow \phi\left(A_{2} u(t)\right),
$$

i.e. $\left\|A_{1} v_{n}(t)\right\| \rightarrow\left\|A_{1} v(t)\right\|$ and $\left\|A_{2} u_{n}(t)\right\| \rightarrow\left\|A_{2} u(t)\right\|$.

Therefore,

$$
\begin{aligned}
\left\|A V_{n}(t)\right\| & =\left\|\left(A_{1} v_{n}(t), A_{2} u_{n}(t)\right)\right\| \\
& =\left\|A_{1} v_{n}(t)\right\|+\left\|A_{2} u_{n}(t)\right\| \\
& \rightarrow\left\|A_{1} v(t)\right\|+\left\|A_{2} u(t)\right\| \\
& \rightarrow\left\|\left(A_{1} v(t), A_{2} u(t)\right)\right\| \\
& \rightarrow\|A V(t)\| .
\end{aligned}
$$

This means that $\phi\left(A V_{n}(t)\right) \rightarrow \phi(A V(t)), \forall \phi \in E^{*}, t \in I$. Thus, $A: \Omega \rightarrow \Omega$ is weakly sequentially continuous.

Since all conditions of Theorem 2 are satisfied, then the operator $A$ has at least one fixed point $(u, v) \in \Omega$, then the coupled system (3)-(4) has at least one weak solution. 


\section{Initial Value Problem}

Consider now the coupled system of the initial value problems (1)-(2)

Theorem 4. Under the assumptions of Theorem 3, the coupled system of the initial value problems (1)-(2) has at least one solution $(x, y) \in X$.

Proof. Let $\frac{d x}{d t}=u \in C[I, E]$ and $\frac{d y}{d t}=v \in C[I, E]$, then

$$
x(t)=x_{0}+\int_{0}^{t} u(s) d s \in C[I, E],
$$

and

$$
y(t)=y_{0}+\int_{0}^{t} v(s) d s \in C[I, E],
$$

are differentiable and $(u(t), v(t))$ will be the solution of the coupled system of the functional equations

$$
\begin{aligned}
& u(t)=f_{1}(t, v(t)), \quad t \in[0, T], \\
& v(t)=f_{2}(t, u(t)), \quad t \in[0, T] .
\end{aligned}
$$

\section{References}

[1] Bashir Ahmad, Juan J. Nieto, Existence results for a coupled system of nonlinear fractional differential equations with three-point boundary conditions, Computers and Mathematics with Applications., 58 (2009), 1838-1843.

[2] J.M. Ball, Weak continuity properties of mapping and semi-groups, Proc. Royal Soc.Edinbourgh Sect., A72 (1973), 275-280.

[3] C. Bai, J. Fang, The existence of a positive solution for a singular coupled system of nonlinear fractional differential equations, Appl. Math. Comput, 150 (2004), 611-621.

[4] Y. Chen, H. An, Numerical solutions of coupled Burgers equations with time and space fractional derivatives, Appl. Math. Comput, 200 (2008), 87-95.

[5] A.M.A. EL-Sayed, H.H.G. Hashem, A coupled systems of fractional order integral equations in reflexive Banach spaces, Commentationes Mathematicae, No.1 (2012) 21-28.

[6] A.M.A. El-Sayed, H.H.G. Hashem, Coupled systems of integral equations in reflexive Banach spaces, Acta Math. Sci 32B(5) (2012), 1-8.

[7] A.M.A. EL-Sayed, H.H.G. Hashem, Coupled systems of Hammerstein and Urysohn integral equations in reflexive Banach spaces, Differential Equation and Control Processes, No.1(2012) 1-12. 
[8] A.M.A. EL-Sayed, W. G. El-Sayed, A. A. H. Abd El-Mowla, On the existence of weakly continuous solutions of the nonlinear functional equations and functional differential equations in reflexive and nonreflexive Banach spaces, Differential Equation and Control Processes. No.1(2016) 32-39.

[9] V. Gafiychuk, B. Datsko, V. Meleshko, Mathematical modeling of time fractional reactiondiffusion systems, J. Comput. Appl. Math. 220 (2008), 215-225.

[10] V.D. Gejji, Positive solutions of a system of non-autonomous fractional differential equations, J. Math. Anal. Appl, 302 (2005), 56-64.

[11] V. Gafiychuk, B. Datsko, V. Meleshko, D. Blackmore, Analysis of the solutions of coupled nonlinear fractional reaction diffusion equations, Chaos Solitons Fract, 41 (2009), 10951104 .

[12] W.J. Knight, Solutions of differential equations in B-spaces, Duke Math. J, 41 (1974), 437-442.

[13] M.P. Lazarevic, Finite time stability analysis of $P D^{\alpha}$ fractional control of robotic timedelay systems, Mech. Res. Comm, 33 (2006), 269-279.

[14] D. O'Regan, Fixed point theory for weakly sequentially continuous mapping, Math. Comput. Modeling. 27 (5)(1998), 1-14.

[15] D. O'Regan, Weak solutions of ordinary differential equations in Banach spaces, Appl.Math. Lett ., 12 (1999), 101-105.

[16] W. Rudin, Functional Analysis, McGraw-Hill, Inc. New york, Harlow(1991).

[17] H. A. H. Salem, A. M. A. El-Sayed, Weak solution for fractional order integral equations in reflexive Banach spaces, Math. Slovaca, 55 No.2(2005), 169-181.

[18] X. Su, Boundary value problem for a coupled system of nonlinear fractional differential equations, Appl. Math. Lett. 22 (2009), 64-69. 